\title{
How quantifiers influence the conceptual representation of plurals
}

\author{
Nikole D. Patson ${ }^{1}$ \\ Accepted: 24 November 2020 / Published online: 7 January 2021 \\ (C) The Psychonomic Society, Inc. 2021
}

\begin{abstract}
This paper reports the results of two experiments that investigate the nature of plural conceptual representations that are created during sentence comprehension. Previous work has found that comprehenders seem to represent both a singular object and a plural set of objects during the comprehension of plural nouns. The activation of the singular object has been attributed to the pragmatic processing involved in understanding the plural (Patson, Journal of Experimental Psychology: Learning, Memory and Cognition, 42, 1140-1153, 2016a). The goal of the current study was to further investigate this hypothesis. Experiment 1 used a picture-matching paradigm to investigate how comprehenders conceptualize plural nouns quantified with many, which renders the scalar implicature unnecessary. Consistent with the pragmatic processing hypothesis, comprehenders did not activate a singular form when the plural was quantified with many. Experiment 2 was designed to further investigate whether all quantifiers block activation of the singular form. The same picture-matching paradigm was used with numerical quantifiers that specify numbers either within or above the subitization range. When the number was within the subitization range, comprehenders' conceptual representations contained exactly that number of objects, and importantly did not contain a singular object. When number was above the subitization range, comprehenders' conceptual representations did not contain an exact number of objects and seemed to activate a singular object. These data are consistent with constraints on how many objects can be represented in visual working memory. Taken together, the results of these two experiments suggest that the plural's conceptual representation emerged as a result of grammatical processing as well as limits on the visual processing system.
\end{abstract}

Keywords Plural $\cdot$ Quantifiers $\cdot$ Language comprehension $\cdot$ Referential processing

In addition to constructing syntactic and semantic representations during language comprehension, comprehenders also construct conceptual mental representations. There has been much debate about the kinds of information included in these representations and the form these representations take (e.g., Barsalou, 1999; Johnson-Laird, 1983). One important area of investigation is the nature of the representation of plural information. The plural is important because plurals are not simply collections of singulars (e.g., Link, 1983), and therefore plural references have properties that can only be attributed to sets, not to individuals (Treisman, 2006). To complicate matters further, as discussed in more detail below, there are a number of grammatical factors that lead to the introduction of plural references (e.g., distributed predicates, pragmatic inferences, quantifiers). A complete understanding of the nature of conceptual representations requires an understanding of how

Nikole D. Patson

Patson.3@osu.edu

1 Ohio State University, 1465 Mount Vernon Avenue, Marion, OH 43302, USA these grammatical processes and "set properties" are instantiated in those representations. While it is clear that comprehenders represent both morphological and conceptual number (e.g., Berent, Pinker, Tzelgov, Bibi, \& Goldfarb, 2005; Humphreys \& Bock, 2005; Kaup, Kelter, \& Habel, 2002; Patson \& Warren, 2010), the nature of and factors that influence those conceptual representations is less clear. There is some evidence to suggest that comprehenders create conceptual representations for plurals that are image-like and contain explicit number information when the number information is within the subitization range (Beg, Čičko, \& Domijan, 2019; Patson, 2016a; Patson, George, \& Warren, 2014; Šetić $\&$ Domijan, 2017). However, it is currently unknown whether the nature of the representations that are generated is due to grammatical processing of the plural or due to constraints on the visual system. The current paper presents two experiments that investigate this issue.

Previous work investigating the conceptual representation of the plural has relied on a picture-matching paradigm. In the picture-matching paradigm, participants read or listen to sentences and then see a picture of an object. Participants' task is to either name the object or decide whether or not the picture 
is of an object described in the sentence. Zwaan, Stanfield, and Yaxley (2002) used this paradigm to test whether comprehenders simulate the action described in sentences. In their experiment, participants read sentences like "The eagle was flying in the sky" or "The eagle was sitting in its nest." Participants were faster to respond to a picture of an eagle when the eagle's shape matched the shape implied by the sentence (e.g., an eagle with its wings spread in the "flying" sentence; an eagle with its wings folded in the "nest" sentence). They used this evidence to support the hypothesis that comprehenders create detailed conceptual representations of objects mentioned in a sentence during comprehension.

As mentioned above, the picture-matching paradigm has been used to investigate the conceptual representation of the plural (Patson, 2016a; Patson et al., 2014; Šetić \& Domijan, 2017). For example, in the first experiment reported in Patson (2016a), participants read sentences that either implied a set of scattered objects (1a) or a set of grouped objects (1b):

(1a) The breeze scattered the leaves.

(1b) The gardener raked up the leaves.

Then participants saw either a picture of a single object, a picture that matched the scattered configuration, or a picture that matched the group configuration (see Fig. 1).

Participants were trained to base their decisions on object identity, not on how many objects were present (i.e., the correct response for all picture types in the experimental condition was "yes"). Participants were faster to accept pictures that matched the spatial configuration of objects implied by the sentence. That is, participants were faster to respond to a picture of a pile of leaves for sentence (1b) compared with a picture of a bunch of scattered leaves. Experiment 2 showed a similar finding for set size: Participants' responses indicated that their representations contained information about relative set size. What was particularly interesting about this study is that participants were also faster to respond to the picture of a single leaf than to the mismatched picture. This was not expected because if participants were building a conceptual representation of the scene described in the sentence, they should have only preferred the image that matched that conceptual representation.

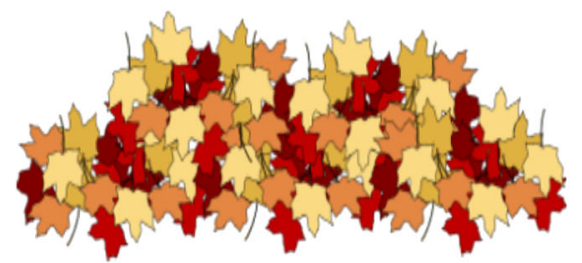

Fig. 1 Stimuli used in Patson (2016a)
The finding that comprehenders seem to activate both a plural and a singular representation when processing the plural was also reported in Patson et al. (2014). Patson (2016a) interpreted the preference for a single picture within a scalar implicature account of the plural. To explain, most semantic accounts of the plural assume that the plural is unmarked for number (Sauerland, Anderssen, \& Yatsushiro, 2005). This assumption is based on the fact that, in some contexts, the plural does not strictly refer to "more than one" object. For example, if someone were to ask, "Do you have cats?," a listener who owns only a single cat could felicitously answer "yes." Their answer would be honest because in this context of the question, cats does not strictly mean "more than one cat." Examples like these suggest that the "more than one" interpretation is not an inherent part of the plural's meaning, therefore not part of its logical semantic definition. However, in most contexts the plural is interpreted as meaning "more than one," so where does this multiplicity inference come from? It has been argued that the "more than one" meaning is derived via a pragmatic inference known as a scalar implicature (Patson, 2016a; Spector, 2007; Tieu, Bill, Romoli, \& Crain, 2014; Zweig, 2009). A scalar implicature is an inference that arises when a weak expression is used instead of a stronger expression. For example, "The coffee is warm" is typically taken to mean that the coffee's temperature is warm, but not hot or scalding. However, "The coffee is warm" does not logically rule out the possibility that the coffee is hot. The fact that comprehenders seem to rule out the possibility that the coffee is hot suggests that comprehenders assume that speakers use the strongest labels that are compatible with their intended meaning and interpret the fact that a weaker expression was used to indicate that the stronger meaning was not appropriate (Grice, 1975). Coming back to the case of the plural, a scalar implicature account assumes that the literal, semantically defined interpretation of the plural is something like "at least one" and an implicature must be made to derive the "more than one" interpretation. There is evidence that the plural behaves similarly to other kinds of scalar implicatures, strengthening the hypothesis that the plural is a form of scalar implicature (e.g., Chierchia, 2004; Levinson, 2000; Tieu, Bill, Romoli, \& Crain, 2014, 2020).
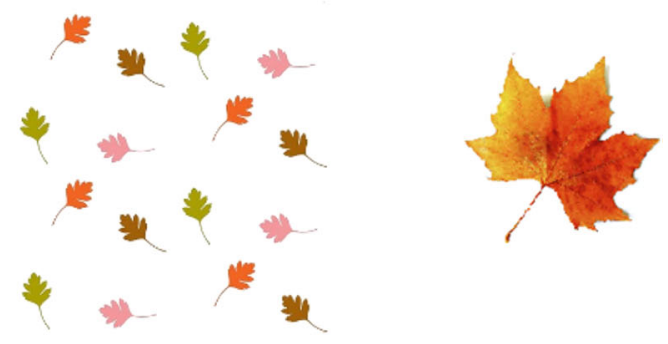
With respect to the picture-matching paradigm, Patson (2016a) argued that participants' picture-judgment times after reading a plural noun were consistent with the scalar implicature hypothesis. Specifically, the activation of the singular representation is consistent with the activation of the semantic meaning of the plural (which includes singular instances), while the activation of the multiple object representation suggested that the pragmatic inference had also been made. This hypothesis is consistent with previous evidence suggesting that comprehenders simulate intermediate grammatical stages of processing. For example, Kaup, Lüdtke, and Zwaan (2006) found that immediately after reading a sentence like "The umbrella was not open," participants responded equally quickly to a picture of an open umbrella as well as a closed umbrella. However, when the sentence was followed by a 1,500-ms delay, participants responded quicker to the picture that represented the actual state of affairs (e.g., the closed umbrella) compared with the object that was negated (e.g., the open umbrella). Kaup et al. (2006) argued that these data suggest that participants first simulated the proposition implied in the sentence (e.g., the umbrella is open) and then negated it. After a brief interval, comprehenders were left with only a representation of the actual state of affairs.

Importantly, the multiplicity interpretation of a plural is not always derived via a scalar implicature. For example, in the sentence, "There are many cats," many specifies that the upcoming plural is to be interpreted as referring to multiple objects, thus, the scalar implicature is blocked because comprehenders no longer need to infer that the plural refers to multiple objects. In the picture-matching paradigm, if the activation of the singular object is due to scalar implicature processing, then there should be no activation of the singular when a quantifier specifies multiplicity. Patson et al. (2014) provided some evidence consistent with this prediction. In their first experiment, participants read sentences like, "There are two apples." Participants were faster to respond to a picture of exactly two apples compared with a picture of only one apple and a picture of more than one apple. Thus, these data suggest that when multiplicity is specified, comprehenders do not activate a singular object. However, currently, it is unknown whether number has to be specified or whether other quantifiers that specify multiplicity have the same effect.

One set of findings suggests that plurals of all set sizes are not represented similarly. Šetić and Domijan (2017) provided evidence consistent with Patson et al. (2014) that comprehenders' conceptual representations have exact number represented. In their picture-matching experiments, participants read sentences like "Three dogs were wandering in the street." Šetić and Domijan found that participants were faster to respond to a picture of dogs when the number matched what was implied in the sentence compared with when it did not. Importantly, participants' responses were based on object identity, not simply a number match —-that is, they were not as fast to respond to a picture of three cats when the sentence described three dogs. However, recent evidence using the picture-matching paradigm found that comprehenders do not simulate exact number when the cardinal quantifier (i.e., six, eight) is outside of the subitization range (Beg et al., 2019). Beg et al. (2019) argued that when numbers are outside of the subitization range, comprehenders do not access a nonsymbolic representation of that number's respective quantity.

Beg et al.'s (2019) findings are consistent with Treisman's (2006) theory of visual attention. Treisman argues that after attention has been directed toward an object, its features (such as color, orientation) are bound together into a single object file that is mentally represented. Treisman points out that visual working memory has a limited capacity and that only three or four object files can be maintained at any given point. Given that our conceptual representations have the same limitations as the visual system (Johnson-Laird, 1983), our conceptual system is likely limited to representing three or four discrete objects (i.e., the subitization range) during sentence processing. However, we are able to comprehend sentences with plural references that refer to more than four objects. This apparent conundrum can be reconciled by returning to Treisman's theory of visual attention. Treisman argues that if a visual scene contains multiple objects of a similar kind, viewers deploy attention more globally over several objects at once and open a single object file for the entire group. Instead of having the same properties as a single object, this object file includes properties of the group as a whole, including global shape, global boundaries, and global relations among the elements. The findings reported in Patson (2016a) are consistent with this theory. Patson found that participants were faster to respond to pictures of multiple objects when the picture matched the spatial configuration suggested in the sentence. For example, participants were faster to respond to a picture of a pile of leaves after reading "The gardener raked up the leaves" compared with a picture of scattered leaves. This suggests that the conceptual representation that they built for the sentence contained properties of the group as a whole, not the individual objects that comprised the group.

There is an important distinction between visual scene comprehension and sentence comprehension. During sentence comprehension, comprehenders must also keep track of the identity of the objects that comprise the group. That is, a pile of green apples and a pile of green balls would have very similar global properties, but the objects comprising the group do not. ${ }^{1}$ Johnson-Laird (1983) suggested that when comprehenders face a large quantity, their mental model

\footnotetext{
${ }^{1}$ Of course, even during visual comprehension, people may need to keep track of what objects comprise a set. However, they can do so by changing the focus of their attention. Rather than deploying attention globally, they can redirect their attention to home in on a single object.
} 
may contain a single token of the objects that comprise the plural to stand in for the group. The proposal presented here is that during the comprehension of sentences with plural referents, comprehenders' representations may contain both a representation of the global properties of the plural set as well as a token of the object that comprises the plural. Specifically, this token representation is required when the number information in the plural is either unspecified or exceeds the capacity of visual working memory (or is outside of the subitization range; Beg et al., 2019; Patson et al., 2014; Šetić \& Domijan, 2017). This could explain the activation of the singular object reported in Patson et al. (2014) and Patson (2016a): Perhaps comprehenders were activating both a representation of the global properties of the plural set as well as a singular token of the objects that comprise the set.

Taken together, there is some ambiguity about the role of the singular representation that is activated when plural nouns are comprehended. It is unclear whether activation of the singular is due to grammatical processing (as argued in Patson, 2016a) or whether limits on the visual processing system lead comprehenders to represent a singular token of the objects that comprise the plural set. The goal of the current study is to investigate the role of quantifiers on the conceptual representation built for plural nouns. Experiment 1 uses a generalized quantifier (i.e., many) that specifies that the upcoming noun refers to more than one object, thus rendering the scalar implicature unnecessary. If the activation of the singular object is due to grammatical processing, it should not be active during the processing of sentences with this quantifier. Under a visual limitations hypothesis, the singular would still be activated even with a generalized quantifier because the number information is unspecified, and therefore the system activates a token. Experiment 2 explores cardinal quantifiers within and outside of the subitization range. If the activation of the singular object is due to grammatical processing, it should not be active during the processing of sentences with these quantifiers because the quantifier renders the scalar implicature unnecessary. Under a visual limitations hypothesis, the singular would be activated only when the cardinal quantifier is outside of the subatization range due to the need of a token representation.

\section{Experiment 1}

The goal of Experiment 1 was to test the grammatical processing account of the plural. If the "two or more" meaning associated with a plural is derived via a scalar implicature, then quantifiers that specify multiplicity block the need for a scalar implicature. If the activation of the singular object during the processing of plural noun phrases is due to scalar implicature processing, then comprehenders should not activate singular representations when reading sentences with quantifiers that render the scalar implicature unnecessary.

\section{Method}

\section{Participants}

Sixty-two volunteers were recruited from the Columbus Center of Science and Industry. All were native speakers of English and had no history of language/hearing disorders. A statistical power analysis was performed for sample size estimation, assuming a medium effect size .06 . With an alpha $=$ .05 and power $=0.95$, the projected sample size needed with this effect size (G*Power 3.1.9.7) is approximately $N=53$. Thus, our actual sample size in this experiment is more than adequate to detect the effects of interest.

\section{Design and stimuli}

Experiment 1 had a $3 \times 2$ repeated-measures design. The first factor was sentence type. The sentence contained either a singular (2a), unquantified plural (2b), or plural quantified by many (2c). The second factor was picture type. The picture either depicted one object or multiple objects.

(2a) The farmer saw an apple.

(2b) The farmer saw apples.

(2c) The farmer saw many apples.

There were 54 experimental items counterbalanced across six lists such that participants saw all six conditions, but only one version of each item. Each list contained the same 54 filler items. Some of the filler items contained quantifiers. All of the filler items required a "no" response.

The pictures used in the current study were a subset of the pictures used in Patson et al. (2014). In order to verify that all three picture types were similarly easy to identify, Patson et al. (2014) conducted a norming study in which participants were shown a label (e.g., leaves) and asked to judge whether a picture was a good match for that label. There were no response-time differences based on picture type, indicating that any response-time differences in the current experiment are not due to differences in how the pictures are visually processed. For the plural pictures, between four and nine instances of the singular object were included in the picture.

\section{Apparatus}

The trials were presented using E-Prime Version 2 experimental software (Schneider, Eschman, \& Zuccolotto, 2002). A Dell P2412H 24-inch monitor $(1,920 \times 1,080$ pixels $)$ displayed stimuli with a screen refresh rate of $60 \mathrm{~Hz}$. 
Keyboard presses were used to log responses and record reaction time.

\section{Procedure}

Participants were tested individually or in pairs. After they provided informed consent, they were given a verbal introduction to the experiment. Then the computer guided them through example trials followed by four practice trials with feedback. As in Patson et al. (2014) and Patson (2016a), participants were instructed and trained (through practice items) to base their judgments on object identity, not the number of objects. That means that for all of the experimental items, the correct answer to the picture judgment is "yes," regardless of how many objects appear in the picture. Each trial began with a left-justified fixation cross. When participants pressed the space bar, the cross was replaced by a sentence. Participants read the sentence at their own pace and pressed the space bar. The sentence disappeared and a picture appeared in the center of the screen. Participants decided whether or not the picture was of an object mentioned in the sentence by pressing "Y" for yes and " $\mathrm{N}$ " for no on the keyboard. The picture disappeared only when participants made their response. The participants' button presses and response times were recorded.

\section{Predictions}

The grammatical processing hypothesis predicts an interaction between sentence and picture conditions. In the plural condition, there should be no reaction-time difference between the match and mismatch conditions, but in the singular and many conditions, participants should be faster to respond to the match pictures than to the mismatch pictures.

The visual limitations hypothesis also predicts an interaction between sentence and picture conditions. However, under this hypothesis there should be no reaction-time difference between the match and mismatch conditions for both the many and plural conditions, indicating that participants have no preference for a singular or plural interpretation of the noun phrase. In the singular condition, participants should be faster to respond to the match pictures than to the mismatch pictures.

\section{Results}

Overall, accuracy on the task was high $(M=95 \%, S D=6 \%)$. One participant was dropped from the analysis due to extremely low accuracy $(M=53 \%)$, and four were dropped for not following directions (i.e., they used number as a criterion for responding to the pictures). The mean reaction times for correct trials are presented in Fig. 2.

Data were subjected to repeated-measures analyses of variance (ANOVAs) using participants (F1) and items (F2) as random factors, and an alpha level of .05 was used for all statistical

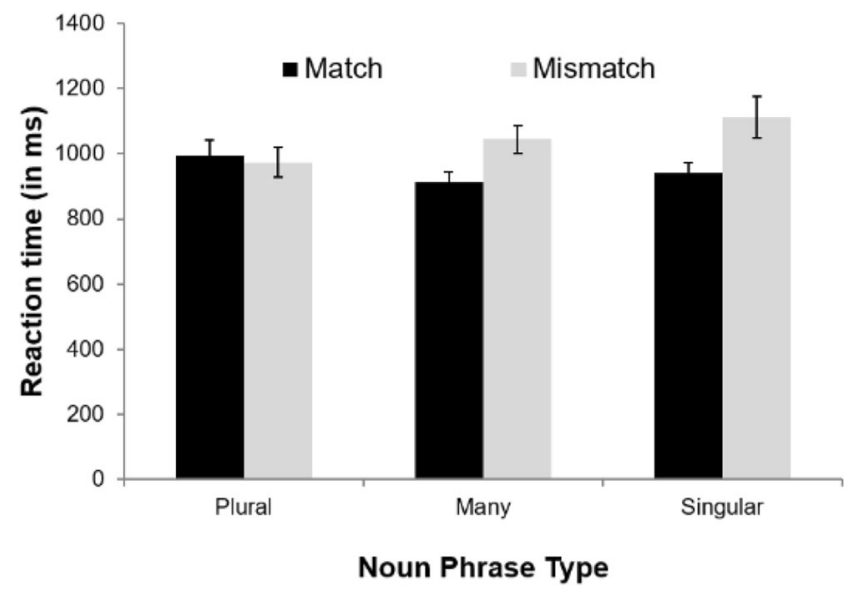

Fig. 2 Experiment 1 mean reaction times (by participants) for correct picture judgments by noun phrase type. Error bars represent \pm 1 standard error

tests. As items were counterbalanced across six lists, the list was included as a between-subjects factor. The list factor did not significantly affect any of the experimental manipulations (all $p s>.05$ ), so it was excluded from the analyses below. The main effect of picture type was significant, $F 1(1,61)=10.09$, $M S E=79874.09, p=.002$, partial $\eta^{2}=.14 ; F 2(1,53)=9.76$, $M S E=42695.82, p=.003$, partial $\eta^{2}=.16$. The main effect of sentence type was not significant, $p>.10$. The main effect of sentence type was qualified by a significant interaction, $F 1(2$, $122)=5.18, M S E=61634.56, p=.007$, partial $\eta^{2}=.08 ; F 2(2$, 106) $=3.54, M S E=50319.57, p=.032$, partial $\eta^{2}=.063$. Planned comparisons indicated that when the critical noun phrase in the sentence was singular or quantified by many, participants were faster to respond accurately to the picture when the picture matched the sentential context than when it did not, many: $t 1(61)=3.44, p=.001$, mean difference $=$ $129.84 \mathrm{~ms} ; t 2(53)=3.27, p=.002$, mean difference $=114.29$ ms; singular: $t 1(61)=3.33, p=.001$, mean difference $=171.04$ $\mathrm{ms} ; t 2(53)=2.82, p=.007$, mean difference $=122.83 \mathrm{~ms}$. However, when the critical noun phrase in the sentence was a plural definite description, there was no difference in reaction time for either picture type, $p \mathrm{~s}>.10$.

\section{Discussion}

The results of Experiment 1 replicated previous findings (Patson, 2016a; Patson et al., 2014): When the sentence had an unquantified plural noun phrase (e.g., apples), participants were equally quick to respond to a picture of a single object and a picture of multiple objects. When the sentence had a singular noun phrase, participants were faster to respond to a picture of a single object compared with a picture of multiple objects.

The novel finding in Experiment 1 is the pattern of data associated with the sentences containing a plural quantified by many. In those sentences, participants were faster to respond to a picture of multiple objects than to a picture of a singular 
object. This finding is consistent with the grammatical processing account of the plural conceptual representation. As discussed above, the quantifier many specifies that the noun phrase refers to multiple objects, thus rendering the scalar implicature unnecessary. Thus, these data suggest that when participants do not need to compute a scalar implicature, they do not activate a singular object.

Although many specifies that the referent refers to more than one object, it is the case that the quantifier's meaning is nonspecific and context-dependent (Partee, 1988). To illustrate, imagine a scenario in which there are 200 sheep on a hill and three of them have red paint on their fleece. A speaker could not felicitously describe that situation by saying "Many of the sheep have red paint on them." However, imagine a scenario where there are exactly five awards given at a ceremony, and one attendee, Hannah, won exactly three of them. In this situation, a speaker could felicitously say, "Hannah won many of the awards last night." These examples point out that while many specifies that there are more than one, it does not invoke a specific quantity, and the quantity that it does invoke is highly context dependent. To account for this, some semantic accounts treat many like a cardinal quantifier that means something like "at least $n$," where $n$ represents a specific number of objects and is determined by the context (Partee, 1988). Other accounts have argued that quantifiers like many do not stand in for specific amounts or numbers (Moxey \& Sanford, 1993, 2000; Nouwen, 2010; Paterson, Filik, \& Moxey, 2009). Importantly, the nature of the conceptual representation of the plural may depend on whether or not number information is specified. Therefore, Experiment 2 was designed to explicitly test whether comprehenders activate a token referent when representing specific numbers of objects outside of the subitization range.

\section{Experiment 2}

Previous work using the picture-matching paradigm has shown that when number is specified using a cardinal quantifier, comprehenders create a conceptual representation that includes exactly that number of objects (Beg et al., 2019; Patson et al., 2014; Šetić \& Domijan, 2017). Importantly, Patson et al. found that when a sentence contained a twoquantified noun phrase, participants did not seem to activate a singular object. That is, they were slower to respond to a singular picture than to a picture of exactly two objects. This finding is consistent with both the grammatical processing hypothesis as well as the visual limitations hypothesis. The quantifier two specifies that the upcoming noun refers to more than one object, rendering the scalar implicature unnecessary. Thus, under this account, activation of a singular is not expected. Additionally, two objects fit well within the constraints of visual working memory (Treisman, 2006) - that is, comprehenders are able to hold up to four object files in memory. Under the visual limitations hypothesis, if the number of objects being simulated fits within the constraints of working memory, the system does not create a token representation of the objects that comprise the plural.

However, recent evidence using the picture-matching paradigm found that comprehenders do not simulate exact number when the cardinal quantifier (i.e., six, eight) is outside of the subitization range (Beg, Čičko, \& Domijan, 2019). This finding is consistent with the visual limitations hypothesis, as the quantities they tested fall well outside of the individuation capacity for visual working memory (Treisman, 2006). However, Beg et al. (2019) did not test comprehenders' responses to singular pictures. Thus, the goal of Experiment 2 is to test the representations created by comprehenders for numbers outside of the subitization range. Under the grammatical processing hypothesis, the singular should not be active because the quantifier eliminates the processing of the scalar implicature. Under the visual limitations hypothesis, when the number of objects being simulated exceeds visual working memory capacity, the singular should be active because the system creates two object files - one for a token representation of the object that comprises the plural and another that contains the statistical properties of the plural set.

\section{Method}

\section{Participants}

Eighty-three volunteers were recruited from COSI. All were native speakers of English and had no history of language/ hearing disorders. A statistical power analysis was performed for sample size estimation, assuming a medium effect size .06 . With an alpha $=.05$ and power $=0.95$, the projected sample size needed with this effect size (G*Power 3.1.9.7) is approximately $N=53$. Thus, our actual sample size in this experiment is more than adequate to detect the effects of interest.

\section{Design and stimuli}

Experiment 2 had a $3 \times 2$ repeated-measures design. The first factor was sentence type. The sentence contained either an unquantified (3a), a two-quantified (3b), or a six-quantified (3c) plural. The second factor was picture type. The picture either matched the number information in the quantifier or was a singular object. In the match condition, for the twoplural sentence, the picture depicted exactly two objects, for the six-plural sentence, the picture depicted exactly six objects, and for the unquantified plural sentence, the picture depicted exactly three objects.

(3a) The farmer saw apples.

(3b) The farmer saw two apples. 
(3c) The farmer saw six apples.

There were 54 experimental items counterbalanced across 6 lists such that participants saw all six conditions, but only one version of each item. Each list contained the same 54 filler items. Some of the filler items contained quantifiers. All of the filler items required a "no" response.

\section{Procedure and apparatus}

The same procedure and apparatus used for Experiment 1 was used in Experiment 2.

\section{Predictions}

The grammatical processing hypothesis predicts an interaction between sentence and picture conditions. In the unquantified plural condition, there should be no reaction-time difference between the match and mismatch conditions, but in both quantified conditions, participants should be faster to respond to the match pictures than to the mismatch pictures.

The visual limitations hypothesis also predicts an interaction between sentence and picture conditions. However, under this hypothesis, there should be no reaction-time difference between the match and mismatch conditions for both the plural condition and the "six" condition, indicating that participants have no preference for a singular or plural interpretation of the noun phrase. In the "two" condition, participants should be faster to respond to the match pictures than to the mismatch pictures.

\section{Results}

Overall, accuracy on the task was high $(M=92 \%, S D=8 \%)$. Seven participants were dropped for not following directions. The mean reaction times for correct trials are presented in Fig. 3.

Data were subjected to repeated-measures ANOVAs using participants (F1) and items (F2) as random factors, and an alpha level of .05 was used for all statistical tests. As items were counterbalanced across six lists, the list was included as a between-subjects factor. The list factor did not significantly affect any of the experimental manipulations (all $p \mathrm{~s}>.05$ ) so it was excluded from the analyses below. The main effect of picture type was significant by subjects, $F 1(2,162)=12.97$, $M S E=66821.79, p=.001$, partial $\eta^{2}=.14 ; F 2(2,106)=3.84$, $M S E=26947.34, p=.055$, partial $\eta^{2}=.07$. The main effect of noun phrase type was not significant, all $p \mathrm{~s}>.10$. The main effect of picture type was qualified by a significant interaction, $F 1(2,162)=10.98, M S E=50802.62, p<.001$, partial $\eta^{2}=.12$; $F 2(2,106)=7.64, M S E=20844.81, p=.001, \operatorname{partial} \eta^{2}=.13$. Planned comparisons indicated that when the critical noun phrase in the sentence was quantified by two, participants were faster to respond accurately to the picture when the picture matched the number of objects in the quantifier than when it

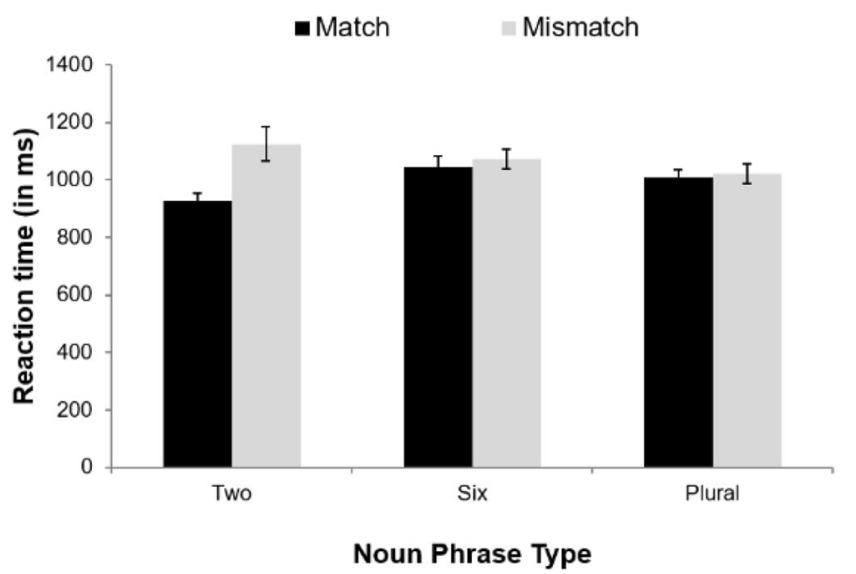

Fig. 3 Experiment 2 mean reaction times (by participants) for correct picture judgments by noun phrase type. Error bars represent \pm 1 standard error

did not, $t 1(82)=4.40, p<.001$, mean difference $=207.44 \mathrm{~ms}$; $t 2(53)=5.40, p<.001$, mean difference $=121.63 \mathrm{~ms}$. However, when the critical noun phrase in the sentence was a plural definite description or quantified by six, there was no difference in reaction time for either picture type, $p \mathrm{~s}>.10$.

\section{Discussion}

The results of Experiment 2 replicated previous findings reported in Patson et al. (2014). When participants read sentences with unquantified plural definite descriptions, they showed no reaction-time preference for pictures containing one object compared with pictures of multiple objects. Additionally, when a sentence contained a plural noun quantified with $t w o$, participants were faster to respond to a picture of exactly two objects compared with a picture of a singular object or a picture of more than two objects.

The novel finding in Experiment 2 is related to participants' picture-judgment times after reading a sentence with a plural noun phrase quantified with six. In these trials, participants were not faster to respond to a picture of exactly six items, consistent with recent data reported by Beg et al. (2019). Instead, participants were equally fast to respond to a picture of a single object as to a picture of multiple objects. This finding is consistent with the visual limitations hypothesis. When the numerical information exceeds visual working memory capacity, comprehenders create a simulation that contains two objects files: one consistent with the plural set and another that represents a token of the objects that comprise the plural.

\section{General discussion}

The two experiments reported in this paper were designed to investigate the nature of plural conceptual representations. The goal of the experiments was to replicate and extend 
previous findings using the picture-matching paradigm. Previous work has shown that when participants read sentences with plural nouns, they activate both a representation of the global set as well as a representation of a singular object that comprises the set (Patson, 2016a; Patson et al., 2014). The goal of these experiments was to begin to understand whether the activation of a singular object was due to grammatical processing related to the plural or due to constraints on the visual system related to conceptualizing plural referents. To compare these hypotheses, two experiments investigated the role of quantifiers on comprehenders' conceptual representations of plural referents.

Experiment 1 was designed to test the nature of the plural representation when computation of the scalar implicature was not necessary in order to derive the multiplicity interpretation of the plural. In Experiment 1, participants read sentences with plural nouns that were quantified by many (e.g., many apples), which specifies that the upcoming noun phrase should be interpreted as referring to "two or more." The results of Experiment 1 showed that when the plural was quantified by many, participants were faster to respond to the picture of multiple objects compared with the picture of a single object. These data are consistent with the grammatical processing hypothesis: When the multiplicity inference is not derived via a scalar implicature, comprehenders do not activate a singular representation. These data are less consistent with the visual processing hypothesis. Under that account, it was predicted that comprehenders would activate a representation that included a global set and a singular object as a token of the objects that comprise the set because quantity information was unspecified.

Experiment 2 tested the effects of two numerical quantifiers on the nature of the plural representation. Previous work has shown that participants tend to be faster responding to pictures that have the same number of objects as mentioned in the sentence when the number of objects is within the subitization range (Beg et al., 2019; Patson et al., 2014; Šetić \& Domijan, 2017). The goal of Experiment 2 was to determine whether number values outside of the subitization range also include a representation of a single object as predicted by the visual processing hypothesis. The results showed that when the noun phrase in the sentence was quantified with $t w o$, participants were faster to respond to a picture of exactly two objects compared with a picture of a single object or a picture of more than two objects. However, when the noun phrase in the sentence was quantified with six, participants were equally fast to respond to a picture of exactly six objects as well as a picture of a single object. These results are consistent with the visual limitations hypothesis which predicts that comprehenders will not activate a singular token when the number of objects depicted is within the subitization range, but will activate a singular token when the set size is outside of the subitization range.
The predictions and observed patterns of data across the two experiments are summarized in Table 1.

Neither the grammatical processing or the visual processing hypothesis perfectly captured the pattern of data reported here. The grammatical processing hypothesis predicted that activation of the singular should disappear whenever the multiplicity interpretation of the plural does not come from a pragmatic inference, such as when a quantifier precedes the noun. While the results of Experiment 1 are consistent with this hypothesis, the results of Experiment 2 are not. In Experiment 2, when the noun was quantified with six the singular was activated. According to the grammatical processing hypothesis, the singular should not have been activated because the scalar implicature was rendered unnecessary via the quantifier.

However, the visual processing hypothesis did not perfectly predict the observed pattern of results either. Under this hypothesis, participants were predicted to activate a singular token anytime quantity information is unspecified (as with the plural and many conditions) or larger than the subatization range (as with six). Although the data reported in Experiment 2 are consistent with this hypothesis, the data reported in Experiment 1 are not. Contrary to the predictions of the visual processing hypothesis, participants did not seem to activate the singular when the noun phrases were quantified by many.

How then can these findings be reconciled? It is possible that during the processing of plural referents, the singular gets activated for multiple reasons. The singular activation could represent both grammatical processing as well as limits on the visual system, and which of these factors is playing the biggest role in the construction of the mental model may be due to the specific type of plural reference. First, the singular seems to be activated when a plural reference is constructed through grammatical processing. In addition to scalar implicature, previous work has found that the singular is activated when a plural reference is derived through distributivity. Patson et al. (2014) found that participants treated singular noun phrases (e.g., box) as plural when they were embedded in distributive predicates (e.g., Each of the men carried a box.). That is, participants were equally fast to respond to a picture of a singular object as to a picture of multiple objects. In the case

Table 1 Predicted and observed rates of activation of the singular

\begin{tabular}{llll}
\hline & GP & VP & Observed \\
\hline Singular (cat) & Yes & Yes & Yes \\
Plural (cats) & Yes & Yes & Yes \\
Many & No & Yes & No \\
Two & No & No & No \\
Six & No & Yes & Yes \\
\hline
\end{tabular}


of distributed predicates, the multiplicity interpretation does not come from a pragmatic inference - thus, the activation of the singular cannot be due to the processing of a scalar implicature. However, it is possible that that activation is due to the grammatical processing of distributivity. As mentioned above, previous work on negation has shown that comprehenders' conceptual representations include representations of intermediate grammatical stages (Kaup et al., 2006). Because some plural referents are derived via grammatical processing that requires activation of singular forms (e.g., distributivity, scalar implicature), it would be consistent with Kaup et al.'s (2006) conclusions that the singular form would get activated during the computation of the plural referent. One inconsistency is that Kaup et al.'s account would predict that singular activation would dissipate over time, as they found that only the actual state of affairs was active after a 1,500-ms delay, not the negated state of affairs. However, Patson (2016b) found that the singular activation after reading a plural noun did not diminish after a 1,500-ms delay. An important difference between Patson (2016b) and Kaup et al. could affect the interpretation of Patson's data. Kaup et al. were investigating negation. In their study, participants activated two representations that were logically incompatible (e.g., an open umbrella and a closed umbrella). Perhaps the deactivation of the initial representation is because it interferes with a representation of the actual state of affairs (one cannot hold a visual representation of both an open and a closed umbrella). This may not be true of the plural. The representation of the singular object may not be visually incompatible with a representation of a set of multiple objects. ${ }^{2}$ Because these two representations (singular and plural) do not interfere with each other, perhaps the activation of the singular does not dissipate over time. This argument is similar to arguments about the differences between ambiguity and vagueness (Frazier \& Rayner, 1990). When words are ambiguous (meaning they map on to two very different meanings; e.g., BUG, referring to an insect or a spying device), comprehenders seem to select one meaning rendering the other meaning inaccessible. However, when words are vague, meaning they map on to multiple related meanings (e.g., NEWSPAPER, referring to the physical paper or to the contents of the paper), participants seem to have a fuzzy representation that allows both related meanings to be partially activated. The idea is that the sentence processor inhibits representations that interfere with maintaining a coherent representation of the discourse. Unlike with negation, although the singular may be activated when comprehending a plural due to grammatical processing,

\footnotetext{
$\overline{2}$ Importantly, I am making an argument about the compatibility of visual representations, not linguistic representations. Under some linguistic accounts, the plural's alternative is assumed to be strengthened to "exactly one." Logically, "exactly one" and "two or more" are inconsistent; however, it is not clear that visually one apple is inconsistent with a picture of two apples in the same way that an open umbrella and a closed umbrella are inconsistent.
}

the singular may not interfere with maintaining a coherent representation, therefore, it is not inhibited.

Second, in cases with cardinal quantifiers, singular activation seems to be more related to constraints on the visual system. It is clear that cardinal quantifiers within the subitization range (e.g., two) instantiate conceptual representations in which exactly that number of discrete objects are present without activating a singular representation. However, when the number is outside of the subitization range, or unspecified, comprehenders seem to activate a singular representation as predicted by the visual processing hypothesis. This is consistent with previously published data showing exact number is included in the representation when it falls within the subitization range (Beg et al., 2019; Patson et al., 2014; Šetić $\&$ Domijan, 2017). These data are consistent with many studies in the number processing literature that show that small numbers (one to four) are processed via a different numerical system than large numbers (e.g., Dehaene, 1997; Trick \& Pylyshyn, 1994).

Finally, conceptual representations built for generalized quantifiers (e.g., many, some, few) may also be more guided by the constraints of the visual system than the grammatical processing system. However, it appears that they do not activate a token representation as initially hypothesized. Instead of a token, comprehenders seem to activate a small set of individuated objects to represent the quantified reference. It is important to note that previous investigations into the usage of generalized quantifiers have shown that these expressions do not simply stand in for particular amounts, number, or proportions. Instead, these quantifiers tend to be thought of as denoting probability distributions that are mentally organized on an ordered but nonlinear scale (Moxey \& Sanford, 1993, 2000; Nouwen, 2010; Paterson et al., 2009; Pezzelle, Bernardi, \& Piazza, 2018). This mean that many may not always refer to set sizes well outside of the subitization range, as was initially assumed. Instead, when a plural is quantified with a generalized quantifier, comprehenders may build a conceptual representation that contains a small set of objects to represent the quantified expression. Thus, the set of objects in Experiment 1's pictures may have matched the conceptual representation created by the comprehenders. This is consistent with work in the reasoning literature showing that when asked to draw their models of quantified expressions, people tend to draw a small set of objects to stand in for the quantified expression (e.g., Bucciarelli \& Johnson-Laird, 1999), and that constraints on visual working memory are one of the factors that influence how many individual objects are included in reasoners' mental models (e.g., Khemlani, Lotstein, Trafton, \& Johnson-Laird, 2015). Importantly, the number of objects in the plural pictures in Experiment 1 was usually outside of the subitization range. However, in their computational model of reasoning with quantifiers, Khemlani et al. (2015) assumed that the number of objects included in a reasoner's mental 
model for quantified sets could be anywhere between two and 10. (According to their model, the set size varies according to a discrete Poisson distribution. The details of this distribution are beyond the scope of this work.) It is possible that the conceptual representation of generalized quantifiers does not include explicit quantity information since that information is not made explicit in the expression. Instead, quantity may be represented as something like "at least two," and pictures that can be quickly matched to that meaning are preferred to those that cannot (i.e., singular). This is consistent with how a plural definite description may be conceptualized. In Patson et al. (2014), the plural pictures contained anywhere from three to six objects. There was no evidence that participants preferred one set size over the other. Taken together, these findings suggest that for plurals where the quantity information is not specified, quantity may not be as explicitly represented as other features, such as an object's shape, orientation, and color (e.g., Mannaert, Dijkstra, \& Zwaan, 2017; Zwaan \& Pecher, 2012; Zwaan et al., 2002).

This revised hypothesis for the representation of generalized quantifiers is consistent with the literature on reasoning with these quantifiers (e.g., Khemlani et al., 2015; Ragni, Khemlani, \& Johnson-Laird, 2014). When presented with quantified expressions, comprehenders often immediately make inferences based on the quantifier. For example, imagine you are told that "none of the students is wearing a red shirt"; you might skip over anyone in a red shirt when counting the number of students in a classroom. In this example, you have inferred that none of the students is wearing a red shirt, therefore, none of the people wearing a red shirt is a student. The theory of mental models explains how comprehenders who are untrained in logic are able to make these kinds of inferences. According to the mental models approach to reasoning, people reason about quantified expressions by first building a mental model of those expressions (e.g., Johnson-Laird, 2006, 2010; Johnson-Laird \& Khemlani, 2013). The mental model theory of reasoning posits that individuals construct iconic models of the situation described in a sentence. So, in order to decide whether the quantified expression "Some of the dogs are brown" is consistent with the expression "All of the dogs are brown," individuals construct a model of each expression and then compare the two models to see if they correspond. This means, then, that reasoning from quantified expressions is a "mental simulation rather than formal manipulation" (Khemlani et al., 2015, p. 2093). While a full discussion of this literature goes beyond the scope of this paper, the mental models theory has shown that these kinds of inferences are available immediately (Khemlani et al., 2015) and lead to predictable illusions of consistency (Kunze, Khemlani, Lotstein, \& Johnson-Laird, 2010). While participants in the current study were not asked to reason from quantified expressions, the data reported here are consistent with these studies in that the current experiments indicate that participants do build iconic mental models of quantified expressions. What the data reported here suggest is that using this experimental paradigm with the same setup, other generalized quantifier (e.g., some, few, most, all) should behave similarly to the many conditions in this study. That is, participants should prefer a picture with multiple objects compared with a picture of a single object, regardless of the quantifier's place on the ordered scale.

Returning to the larger questions regarding the nature of conceptual representations during sentence comprehension, the picture-matching paradigm has been used to suggest that the conceptual representations are image-like and grounded in experience (e.g., Mannaert et al., 2017; Zwaan \& Pecher, 2012; Zwaan et al., 2002). The data reported here and elsewhere (Patson, 2016a, 2016b; Patson et al., 2014) using the picture-matching paradigm to probe the plural conceptual representation add to this body of work. Unlike previous work, data from investigating the plural suggest that conceptual representations built during intermediate stages of grammatical processing may linger especially if they do not interfere with the processor's ability to maintain a coherent representation of the discourse. Additionally, these data may also suggest that for some abstract concepts, like plurality, comprehenders' representations may include token-like representations (see Johnson-Laird, 1983). This is consistent with Zwaan's (2014) argument that some linguistic contexts may require more abstract kinds of representations. The plural is an interesting context in which to begin probing because there are a number of different ways in which a plural reference can be introduced into the discourse (for a discussion see Patson, 2014). Understanding how these different references influence conceptual representations can help elucidate the nature of conceptual representations in general.

The proposal that conceptual representations are related to the object file representations created during visual processing also raise a number of interesting questions about the nature of plural representations. Treisman (2006) argues that visual working memory has a limited capacity and that only three or four object files can be maintained at any given point. Thus, if a visual scene contains multiple objects of a similar kind, viewers deploy attention more globally over several objects at once and open a single object file for the entire group. Instead of having the same properties as a single object, this object file includes properties of the group as a whole, including global shape, global boundaries, and global relations among the elements. Previous work suggests that relative set size and the distribution of objects are included in simulations (Patson, 2016a). However, it is unknown whether properties such as color are included in plural simulations and if so, how that information is simulated. Previous work on single objects provided evidence that color is simulated as part of the object representation (Mannaert et al., 2017; Zwaan \& Pecher, 2012; but cf. Connell, 2007). Following Treisman's proposal, 
properties such as color are likely represented statistically (e.g., $80 \%$ of the set of apples is red). Future work will be necessary to determine how properties such as color are included in plural simulations.

Acknowledgments I'd like to thank Isabel Aey, Meredith Coleman, Chelsea Morgan, Francesca Napoli, Raquel Rodriguez-Ocasio, and Joshua Sarver for their assistance with stimuli creation and data collection. These data were collected at the Language Sciences Laboratory at the Center for Science and Industry (COSI) in Columbus, Ohio.

Open practices statement All of the data reported here are available upon request. Stimuli are available on the Open Science Framework doi:10.17605/OSF.IO/VCA26). None of the experiments was preregistered.

\section{References}

Barsalou, L. W. (1999). Perceptual symbol systems. Behavioral and Brain Sciences, 22, 577-660.

Beg, M. Š., Čičko, J., \& Domijan, D. (2019). Symbol grounding of number words in the subitization range. Psychological Research. https://doi.org/10.1007/s00426-019-01265-4

Berent, I., Pinker, S., Tzelgov, J., Bibi, U., \& Goldfarb, L. (2005). Computation of semantic number from morphological information. Journal of Memory and Language, 53, 342-358.

Bucciarelli, M., \& Johnson-Laird, P. N. (1999). Strategies in syllogistic reasoning. Cognitive Science, 23, 247-303.

Chierchia, G. (2004). Scalar implicatures, polarity phenomena, and the syntax/pragmatics interface. In A. Belletti (Ed.), Structures and beyond (pp. 39-103). Oxford: Oxford University Press.

Connell, L. (2007). Representing object colour in language comprehension. Cognition, 102(3), 476-485.

Dehaene, S. (1997). The number sense. New York: Oxford University Press.

Frazier, L., \& Rayner, K. (1990). Taking on semantic commitments: Processing multiple meanings vs. multiple senses. Journal of Memory and Language, 29(2), 181-200.

Grice, H. P. (1975). Logic and conversation. Syntax and Semantics, 3, $41-58$.

Humphreys, K. R., \& Bock, K. (2005). Notional number agreement in English. Psychonomic Bulletin \& Review, 12, 689-695.

Johnson-Laird, P. N. (1983). Mental models. Cambridge: Cambridge University Press.

Johnson-Laird, P. N. (2006). How we reason. New York: Oxford University Press.

Johnson-Laird, P. N. (2010). Mental models and human reasoning. Proceedings of the National Academy of Sciences of the United States of America, 107(43), 18243-18250.

Johnson-Laird, P. N., \& Khemlani, S. S. (2013). Toward a unified theory of reasoning. In B. Ross (Ed.), Psychology of learning and motivation (Vol. 59, pp. 1-42). New York: Elsevier.

Kaup, B., Kelter, S., \& Habel, C. (2002). Representing referents of plural expressions and resolving plural anaphors. Language and Cognitive Processes, 17(4), 405-450.

Kaup, B., Lüdtke, J., \& Zwaan, R. A. (2006). Processing negated sentences with contradictory predicates: Is a door that is not open mentally closed? Journal of Pragmatics, 38(7), 1033-1050.

Khemlani, S., Lotstein, M., Trafton, J. G., \& Johnson-Laird, P. N. (2015). Immediate inferences from quantified assertions. Quarterly Journal of Experimental Psychology, 68(10), 2073-2096.
Kunze, N., Khemlani, S., Lotstein, M., \& Johnson-Laird, P. (2010). Illusions of consistency in quantified assertions. Proceedings of the Annual Meeting of the Cognitive Science Society, 32(32).

Levinson, S. (2000). Presumptive meanings. Cambridge: MIT Press.

Link, G. (1983). The logical analysis of plurals and mass terms: A latticetheoretical approach. In C. Schwarze, R. Bauerle, \& A. von Stechow (Eds.), Meaning, use, and the interpretation of language (pp. 302 323). Berlin: De Gruyter.

Mannaert, L. N. H., Dijkstra, K., \& Zwaan, R. A. (2017). Is color an integral part of a rich mental simulation? Memory \& Cognition, 45(6), 974-982.

Moxey, L. M., \& Sanford, A. J. (1993). Communicating quantities: A psychological perspective. Mahwah: Erlbaum.

Moxey, L. M., \& Sanford, A. J. (2000). Communicating quantities: A review of psycholinguistic evidence of how expressions determine perspectives. Applied Cognitive Psychology: The Official Journal of the Society for Applied Research in Memory and Cognition, 14(3), 237-255.

Nouwen, R. (2010). Two kinds of modified numerals. Semantics and Pragmatics, 3, 3-1.

Partee, B. (1988). Many quantifiers. Proceedings of ESCOL, 5, 383-402.

Paterson, K. B., Filik, R., \& Moxey, L. M. (2009). Quantifiers and discourse processing. Language and Linguistics Compass, 3(6), 1390 1402.

Patson, N. D. (2014). The processing of plural expressions. Language and Linguistics Compass, 8, 319-329.

Patson, N. D. (2016a). Evidence in support of a scalar implicature account of plurality. Journal of Experimental Psychology: Learning, Memory and Cognition, 42, 1140-1153.

Patson, N. D. (2016b). Singular interpretations linger during the processing of plural noun phrases. In A. Papafragou, D. Grodner, D. Mirman, \& J. C. Trueswell, (Eds.), Proceedings of the 38th Annual Conference of the Cognitive Science Society (pp. 11881192). Austin, TX: Cognitive Science Society.

Patson, N. D., George, G. E., \& Warren, T. (2014). The conceptual representation of number. Quarterly Journal of Experimental Psychology, 67, 1349-1365.

Patson, N. D., \& Warren, T. (2010). Evidence for distributivity effects in comprehension. Journal of Experimental Psychology: Learning, Memory and Cognition, 36, 782-789.

Pezzelle, S., Bernardi, R., \& Piazza, M. (2018). Probing the mental representation of quantifiers. Cognition, 181, 117-126.

Ragni, M., Khemlani, S., \& Johnson-Laird, P. N. (2014). The evaluation of the consistency of quantified assertions. Memory \& Cognition, 42(1), 53-66.

Sauerland, U., Anderssen, J., \& Yatsushiro, K. (2005). The plural is semantically unmarked. In S. Kepser \& M. Reis (Eds.), Linguistic evidence: Empirical, theoretical, and computational perspectives (pp. 413-434). Berlin: De Gruyter. https://doi.org/10.1515/ 9783110197549

Schneider, W., Eschman, A., \& Zuccolotto, A. (2002). E-Prime user's guide. Pittsburgh: Psychology Software Tools.

Šetić, M., \& Domijan, D. (2017). Numerical congruency effect in the sentence-picture verification task. Experimental Psychology, 64, 159-169.

Spector, B. (2007). Aspects of the pragmatics of plural morphology: On higher-order implicatures. In U. Sauerland \& P. Stateva (Eds.), Presupposition and implicature in compositional semantics (pp. 243-281). London: Palgrave Macmillan.

Tieu, L., Bill, C., Romoli, J., \& Crain, S. (2014). Plurality inferences are scalar implicatures: Evidence from acquisition. Semantics and Linguistic Theory, 24, 122-136.

Tieu, L., Bill, C., Romoli, J., \& Crain, S. (2020). Testing theories of plural meanings. Cognition, 203.

Treisman, A. (2006). How the deployment of attention determines what we see. Visual Cognition, 14, 411-443. 
Trick, L. M., \& Pylyshyn, Z. W. (1994). Why are small and large numbers enumerated differently? A limited-capacity preattentive stage in vision. Psychological Review, 101, 80-102.

Zwaan, R. A. (2014). Embodiment and language comprehension: Reframing the discussion. Trends in Cognitive Sciences, 18(5), 229-234.

Zwaan, R. A., \& Pecher, D. (2012). Revisiting mental simulation in language comprehension: Six replication attempts. PLOS ONE, $7(12)$, e51382.
Zwaan, R. A., Stanfield, R. A., \& Yaxley, R. H. (2002). Language comprehenders mentally represent the shapes of objects. Psychological Science, 13, 168-171.

Zweig, E. (2009). Number-neutral bare plurals and the multiplicity implicature. Linguistics and Philosophy, 32(4), 353-407.

Publisher's note Springer Nature remains neutral with regard to jurisdictional claims in published maps and institutional affiliations. 